\title{
Failure of Repeated MDA with Albendazole for Trichuriasis Control in Schoolchildren of the Yangon Region, Myanmar
}

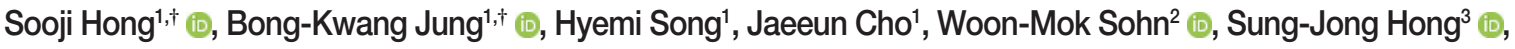 \\ Jin-Youp Ryu', Hyun-Seung Kim ${ }^{4}$, Mun-Hyoo Jeong ${ }^{4}$, Thi Thi Htoon ${ }^{5}$, Htay Htay Tin ${ }^{5}$, Jong-Yil Chai' ${ }^{1,6, *}$ (D) \\ 'Institute of Parasitic Diseases, Korea Association of Health Promotion, Seoul 07649, Korea; ${ }^{2}$ Department of Parasitology and Tropical Medicine, \\ and Institute of Health Sciences, Gyeongsang National University School of Medicine, Jinju 52727, Korea; ${ }^{3}$ Convergence Research Center for Insect \\ Vectors, Incheon University, Incheon 22012, Korea; ${ }^{4}$ Bureau of Health Examination and Management, Korea Association of Health Promotion, Seoul

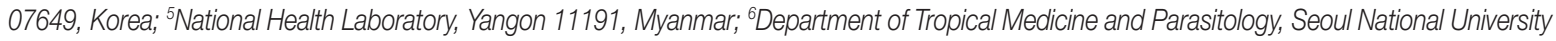 \\ College of Medicine, Seoul 03080, Korea
}

\begin{abstract}
Soil-transmitted helminth (STH) infections are still a considerable challenge in Myanmar. We undertook a control program for STH infections (especially Trichuris trichiura) among schoolchildren in Myanmar using mass drug administration (MDA) and health education. Around 1,700 schoolchildren from 15 primary schools in 3 suburban districts (Shwe Pyi Thar, Twantay, and Kyauktan) of the Yangon Region were subjected in this study during 2017-2019. All of the schoolchildren in each school were orally administered albendazole ( $400 \mathrm{mg}$ in a single dose) 2, 3, and 4 times a year in 2017, 2018, and 2019, respectively. The results revealed that the egg positive rate of any intestinal helminths (including STH) was reduced from $37.6 \%(649 / 1,724)$ in 2017 to $22.8 \%(352 / 1,542)$ in 2019. The egg positive rate of Ascaris lumbricoides was decreased remarkably from $23.3 \%(402 / 1,724)$ in 2017 to $3.6 \%(56 / 1,542)$ in 2019 . However, that of $T$. trichiura was only slightly reduced from $26.9 \%(464 / 1,724)$ in 2017 to $20.2 \%(312 / 1,542)$ in 2019 . The intensity of infection with $A$. lumbricoides and T. trichiura was both more or less reduced, and the proportion of light infection cases with $A$. lumbricoides and T. trichiura increased from $35.6 \%$ in 2017 to $64.3 \%$ in 2019 and from $70.3 \%$ in 2017 to $81.7 \%$ in 2019 , respectively. The results indicated that repeated MDAs (2-4 times a year for 3 years) using albendazole on schoolchildren in Myanmar failed to control T. trichiura infection. For a successful control of trichuriasis in Myanmar, new MDA strategies, using a modified albendazole regimen (multiple daily doses for 2 or 3 days) or an alternative anthelmintic drug, such as oxantel pamoate, is strongly recommended.
\end{abstract}

Key words: Trichuris trichiura, Ascaris lumbricoides, soil-transmitted helminth, trichuriasis, control, mass drug administration (MDA), Myanmar

\section{INTRODUCTION}

More than 1.5 billion people are infected with soil-transmitted helminths (STH) worldwide, which include Ascaris lumbricoides (roundworms), Trichuris trichiura (whipworms), Necator americanus and Ancylostoma duodenale (hookworms), and Strongyloides stercoralis [1]. Over 267 million preschool-age children and over 568 million school-age children live in STH endemic areas and need treatment and preventive/control interventions [1]. Preventive chemotherapy to high-risk population groups by a large-scale distribution of anthelminthic drugs integrated with environmental sanitation and health ed-

• Received 26 October 2021, revised 19 November 2021, accepted 19 November 2021.

*Corresponding author (cjy@snu.ac.kr)

†These authors contributed equally to this article.

(c) 2021, Korean Society for Parasitology and Tropical Medicine

This is an Open Access article distributed under the terms of the Creative Commons Attribution Non-Commercial License (https://creativecommons.org/licenses/by-nc/4.0) which permits unrestricted non-commercial use, distribution, and reproduction in any medium, provided the original work is properly cited. ucation is a core strategy for STH control [2,3].

In Myanmar, after a baseline survey in 2003, large-scale mass drug administration (MDA) has been implemented using albendazole twice a year to 12.67 million preschool-age children and school-age children by the School and Youth Health Program of the Ministry of Health and Sports [4]. After the national repeated MDAs, the overall prevalence of STH showed a significant reduction from $69.7 \%$ (ascariasis $48.5 \%$, trichuriasis 57.5\%, and hookworm infections 6.5\%) in 2003 to $20.9 \%$ (ascariasis $5.8 \%$, trichuriasis $18.6 \%$, and hookworm infections $0.3 \%$ ) in 2012 [4]. In addition, the proportion of moderate-to-heavy intensity of STH infected cases has been reduced from $18.2 \%$ at baseline to less than $7 \%$ after the national MDAs [4].

However, in some regions like 3 districts of the Yangon Region (South Dagon, North Dagon, and Hlaing-thar-yar), the prevalence of STH infections remained still high, up to $32.5 \%$ among schoolchildren in 2013, despite the national school- 
based MDA programs with health education [5]. It was suggested to be due to a high reinfection rate among the population in this region. In order to provide evidence for this suggestion, Chai et al. [6] performed 1-time MDA in different counties of the same 3 districts targeting a total of 2,227 schoolchildren using albendazole $400 \mathrm{mg}$ in a single dose in 2014-2015, and follow-up fecal examinations were performed 4 months later. The pre-treatment egg positive rate of A. lumbricoides infection was $17.2 \%$, and the intensity of infection was 15,532 in average EPG/person (no. of eggs per gram of feces/person), and those of T. trichiura were 19.4\% and 1,074 EPG/person, respectively [6]. Follow-up fecal examinations performed 4 months later revealed considerable decreases in the prevalence and intensity of $A$. lumbricoides to $8.3 \%$ and 12,429 EPG/person, respectively [6]. However, no significant reduction was obtained in the prevalence and intensity of $T$. trichiura which remained at $18.2 \%$ and $862 \mathrm{EPG} /$ person, respectively [6]. These results suggested necessity of more effective MDA strategies for trichuriasis control around the Yangon Region.

The present study was conducted to establish effective MDA strategies to control STH infections (in particular, trichuriasis) among schoolchildren in Myanmar. The strategies applied were firstly, repeated MDAs $(2,3$, or 4 times a year) for a total of 3 years, and secondly, blanket MDA method (treatment of all participants in a community) rather than selective MDAs (treatment of only egg positive individuals, as was done in [6]). The target areas were 3 districts (Shwe Pyi Thar, Twantay, and Kyauktan) of the Yangon Region (different districts from the previous project [6]), where STH infections were found to be highly prevalent.

\section{MATERIALS AND METHODS}

\section{Surveyed areas}

Myanmar (formerly Burma) is a developing country bordering 5 countries in Southeast Asia with 51.5 million people [7]. The country is divided into 15 administrative states and regions ( 7 states, 7 regions, and 1 union territory) and represents a tropical monsoon climate [7]. The country is still prevalent with STH infections, although large-scale national MDA programs have been implemented by the government using albendazole (400 mg) twice a year targeting schoolchildren. Three districts (Shwe Pyi Thar, Twantay, and Kyauktan) of the Yangon Region were selected for this study (Fig. 1). These dis-

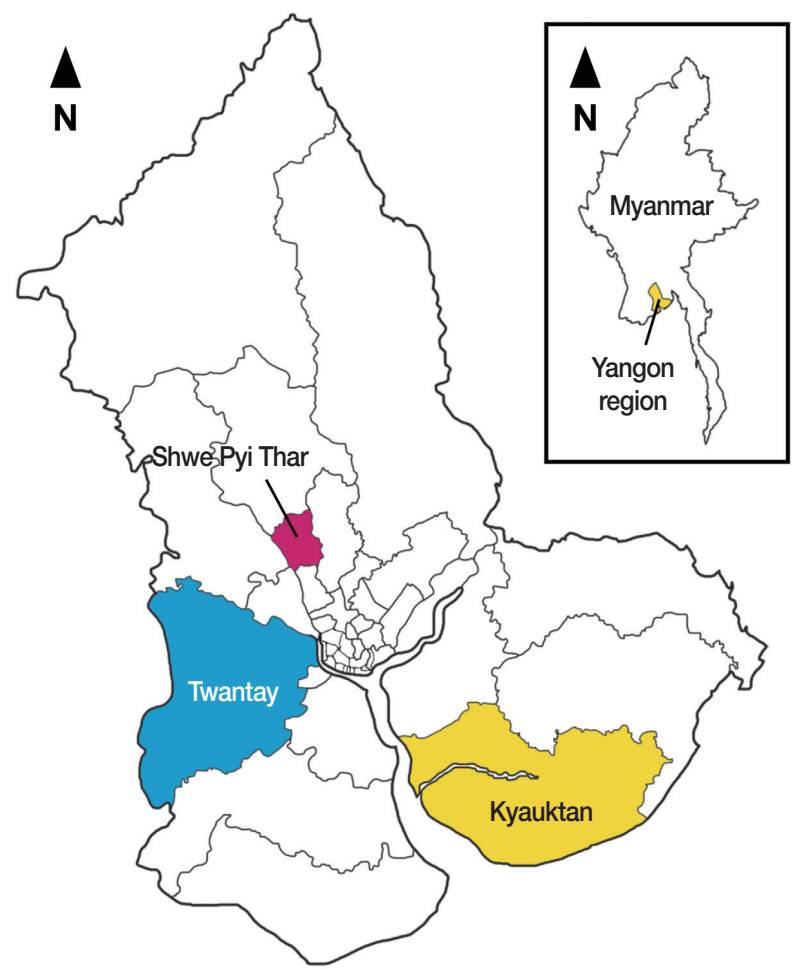

Fig. 1. Map showing the surveyed areas around the Yangon Region, Myanmar. Schoolchildren of 15 primary schools in 3 districts (Shwe Pyi Thar, Twantay, and Kyauktan) were subjected for this study.

tricts are located in the lower reaches of the Yangon River.

\section{MDA and follow-up fecal examinations}

The blanket MDA method was applied targeting all schoolchildren in each primary school irrespective of the fecal egg positivity using a single oral dose of $400 \mathrm{mg}$ albendazole (Burma Pharmaceutical Industry, Yangon, Myanmar). The first MDA was executed in January 2017, and the subsequent MDAs were repeated continually targeting the schoolchildren in the same school, total 9 times until October 2019 which consisted of 2 times in 2017, 3 times in 2018, and 4 times in 2019 (Fig. 2). The coverage rate of each MDA was checked via questionnaires to each schoolchild; the rate was low, $75.1 \%$ in 2017 but it gradually increased to $89.4 \%$ in 2018 and $92.8 \%$ in 2019, which were higher than the WHO's recommendation (minimum of 75\%) [8].

The target population of the first MDA was about 1,800 schoolchildren in 15 primary schools (4 schools in Shwe Pyi Thar, 7 schools in Twantay, and 4 schools in Kyauktan) (Fig. 2). After 5 months (in June 2017), the first follow-up fecal ex- 


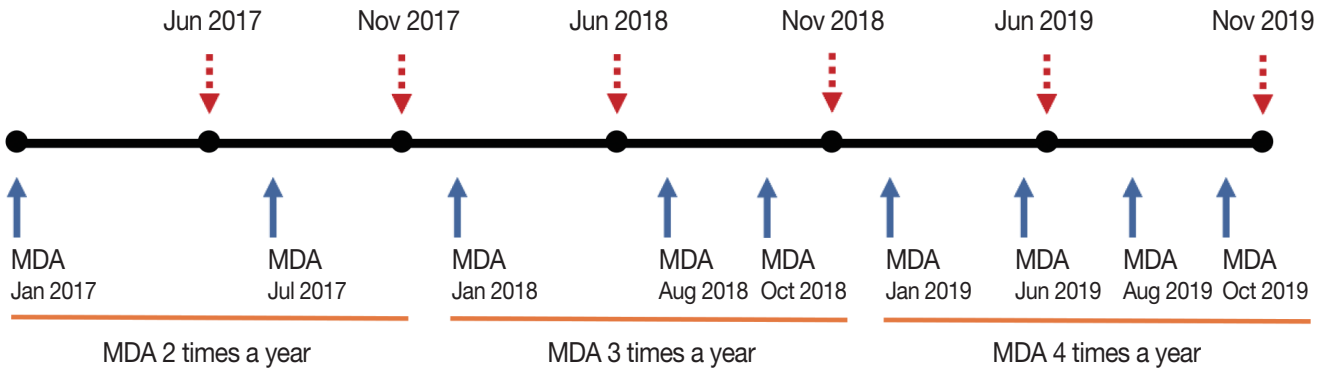

Fig. 2. Scheme of blanket MDA program for STH control in Myanmar, 2017-2019.

amination was performed on a total of 1,724 schoolchildren (mean age, 7.4 years) consisting of almost the same numbers of boys and girls (51:49). In July 2017, the second MDA was executed, and the second follow-up fecal examination was performed in November 2017 on 1,410 schoolchildren (mean age, 7.5 years; gender ratio $\mathrm{M}: \mathrm{F}=53: 47$ ) in 15 primary schools (Fig. 2). The schoolchildren turnover rate was less than 20$25 \%$ between successive follow-up examinations, in other words, about $75-80 \%$ of the schoolchildren remained in the same class between each follow-up examination.

As the egg positive rate of STH, in particular, T. trichiura, revealed no much decrease by the 2 times' MDAs in 2017, the frequency of MDAs was increased to 3 times in 2018 and 4 times in 2019 (Fig. 2). Follow-up monitoring surveys were performed 2 times a year in 2018 and 2019 (Fig. 2). In June 2018, a total of 1,743 schoolchildren (mean age, 7.0 years; gender ratio $\mathrm{M}: \mathrm{F}=52: 48$ ) were examined, and in November 2018, a total of 1,635 schoolchildren (mean age, 7.0 years; gender ratio $\mathrm{M}: \mathrm{F}=49: 51$ ) were examined. In June 2019, a total of 1,702 schoolchildren (mean age, 6.8 years; gender ratio $\mathrm{M}: \mathrm{F}=49: 51$ ) were examined, and in November 2019, a total of 1,542 schoolchildren (mean age, 8.0 years; gender ratio $\mathrm{M}: \mathrm{F}=50: 50$ ) were examined.

\section{Collection of fecal samples and examination}

Fecal samples were collected from each child in primary schools and transported to the National Health Laboratory (NHL) in Yangon, Myanmar. The samples were examined for the eggs of intestinal helminths using the Kato-Katz thick smear method, which is useful for a large-scale STH monitor- ing [2]. One slide per child was prepared and examined under a microscope in the NHL. The EPG was measured by counting the number of helminth eggs found on each Kato-Katz fecal smear multiplied by 24 . The intensity of $A$. lumbricoides and $T$. trichiura infection was divided into 3 categories according to the recommendations by WHO [8]. Light, moderate, and heavy infections for A. lumbricoides were set at 1-4,999, 5,00049,999, and $\geq 50,000 \mathrm{EPG}$ and those for T. trichiura were 1-999, 1,000-9,999, and $\geq 10,000$ EPG, respectively.

\section{Ethical consideration and data processing}

This study was ethically approved by Ethics Review Committee, the Department of Medical Research (DMR, Yangon), Ministry of Health and Sports, Myanmar (ERC no. 005117). The staff of NHL informed of and oriented on the purpose of this project to each school and each participant. Then, the written consent was obtained from guardians of the schoolchildren in the presence of the township medical officer (TMO) and the representative of each school. The data collected from 6 follow-up fecal surveys were processed and analyzed using Excel 2016 (Microsoft, Redmond, Washington, USA) and were statistically evaluated using the chi-square test. The level of statistical significance was set at $P<0.05$.

\section{RESULTS}

In the first follow-up fecal examination in June 2017, the overall helminth egg positive rate (no. examined, 1,724) was $37.6 \%$ (28.9\% in Shwe Pyi Thar, 52.8\% in Twantay, and 17.3\% in Kyauktan), and the average egg positive rate of A. lumbricoi- 
Table 1. Follow-up egg positive rates of intestinal helminths among schoolchildren in 3 districts of the Yangon Region, Myanmar, during the 3-years of MDAs (2017-2019)

\begin{tabular}{lcccccc}
\hline Year & Jun. 2017 & Nov. 2017 & Jun. 2018 & Nov. 2018 & Jun. 2019 & Nov. 2019 \\
\hline No. examined & 1,724 & 1,410 & 1,743 & 1,635 & 1,702 & 1,542 \\
No. of egg positive cases (egg positive rate) & & & & & & \\
Any helminth & $649(37.6)$ & $500(35.5)$ & $624(35.8)$ & $378(23.1)$ & $348(20.4)$ & $352(22.8)$ \\
Ascaris lumbricoides & $402(23.3)$ & $244(17.3)$ & $301(17.3)$ & $47(2.9)$ & $123(7.2)$ & $56(3.6)$ \\
Trichuris trichiura & $464(26.9)$ & $370(26.2)$ & $477(27.4)$ & $348(21.3)$ & $248(14.5)$ & $312(20.2)$ \\
Hookworms & $2(0.1)$ & $11(0.8)$ & $6(0.3)$ & $0(0.0)$ & $4(0.2)$ & $1(0.1)$ \\
Opisthorchis viverrini/MIFa & $5(0.3)$ & $6(0.4)$ & $9(0.5)$ & $6(0.4)$ & $7(0.4)$ & $5(0.3)$ \\
Enterobius vermicularis & $24(1.4)$ & $13(0.9)$ & $31(1.8)$ & $8(0.5)$ & $26(1.5)$ & $18(1.2)$ \\
Taenia spp. & $2(0.1)$ & $0(0.0)$ & $1(0.1)$ & $0(0.0)$ & $2(0.1)$ & $1(0.1)$ \\
Hymenolepis nana & $1(0.1)$ & $2(0.1)$ & $2(0.1)$ & $0(0.0)$ & $4(0.2)$ & $2(0.1)$ \\
Echinostoma sp. & $0(0.0)$ & $0(0.0)$ & $1(0.1)$ & $0(0.0)$ & $0(0.0)$ & $0(0.0)$ \\
Others ${ }^{b}$ & $3(0.2)$ & $1(0.1)$ & $2(0.1)$ & $4(0.2)$ & $4(0.2)$ & $1(0.1)$ \\
\hline
\end{tabular}

${ }^{a} \mathrm{MIF}$, minute intestinal flukes, including heterophyids.

${ }^{b}$ Others include dicrocoelid eggs.

des and T. trichiura was $23.3 \%$ and $26.9 \%$, respectively (Table 1). The egg positive rate of hookworms was $0.1 \%$, and those of other helminths were as follows: Enterobius vermicularis $1.4 \%$, Opisthorchis viverrini $0.3 \%$, Taenia spp. 0.1\%, Hymenolepis nana $0.1 \%$, and others $0.2 \%$ (Table 1 ). Including the first fecal survey, total 6 rounds of follow-up fecal surveys were performed (in June and November in 2017, 2018, and 2019) with the number of schoolchildren examined in each survey ranging from 1,410 to 1,743 (Table 1).

The overall follow-up results revealed that the egg positive rate of any helminths decreased from av. 37.6\% in June 2017 to av. 22.8\% in November 2019 (Table 1; Supplementary Fig. S1). Similar decreasing patterns were observed in 3 districts. The prevalence of any helminths was not significantly different between boys and girls $(P>0.05)$ (data not shown). After repeated MDAs, the egg positive rate of A. lumbricoides was remarkably decreased from $23.3 \%$ in June 2017 to $3.6 \%$ in November $2019(P<0.05)$ (Table 1; Fig. 3A). However, the egg positive rate of $T$. trichiura was only slightly decreased from 26.9\% in June 2017 to 20.2\% in November 2019 (Table 1; Fig. $3 \mathrm{~B})$. Among the 3 districts, Twantay revealed the highest prevalence of A. lumbricoides, followed by Shwe Pyi Thar and Kyauktan. In the final follow-up, the prevalence of A. lumbricoides was within the range 1.4-4.9\% in the 3 districts (Fig. 3A). With regard to T. trichiura, the highest prevalence was also noted in Twantay district, followed by Shwe Pyi Thar and Kyauktan districts; in the final follow-up, the prevalence of T. trichiura remained at $10.1-28.8 \%$ (Fig. 3B).

The average EPG of A. lumbricoides in the first follow-up ex- amination was 1,824 in June 2017, which was decreased to 699 in November 2017 (increased to 1,603 in June 2018), to 491 in November 2018, to 536 in June 2019, and to 481 in November 2019. However, the average EPG of T. trichiura, which was 63 in June 2017, revealed only slight decreases to 68,55 , 39,52 , and 38 in the successive follow-up examinations.

To estimate the decreasing patterns of the intensity of infection, the proportions of schoolchildren with light, moderate, and heavy infections [8] were calculated and presented in Fig. 3C and D. As to A. lumbricoides infection, remarkable changes in the proportion of light infections with A. lumbricoides were observed through 6 follow-up studies (Fig. 3C, D). It was $35.6 \%$ in June 2017 which was elevated to $64.3 \%$ in November 2019, whereas that of moderate and heavy infection cases was significantly decreased from $64.4 \%$ in June 2017 to $35.7 \%$ in November $2019(P<0.05)$ (Fig. 3C, D). In the case of $T$. trichiura infection, the proportion of light infections was 70.3\% in June 2017 which was elevated to $81.7 \%$, whereas that of moderate and heavy infections was significantly reduced from 29.7\% in June 2017 to 18.3\% in November $2019(P<0.05)$ (Fig. 3C, D).

\section{DISCUSSION}

For the control of STH infections, WHO recommended establishing the frequency of MDAs according to the prevalence in each endemic community; MDA once a year for the prevalence of 20-50\%, and MDA twice a year for the prevalence of $\geq 50 \%$ [8]. In our previous study [6], the baseline prevalence 
A

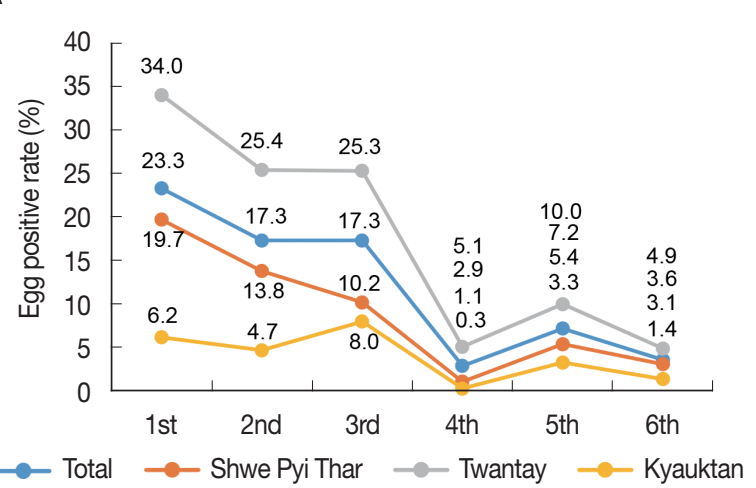

C

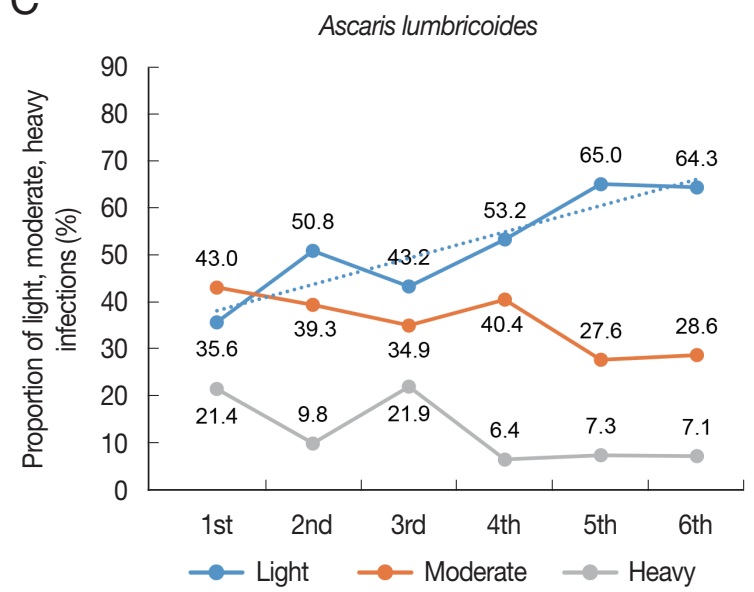

$\mathrm{B}$

Trichuris trichiura

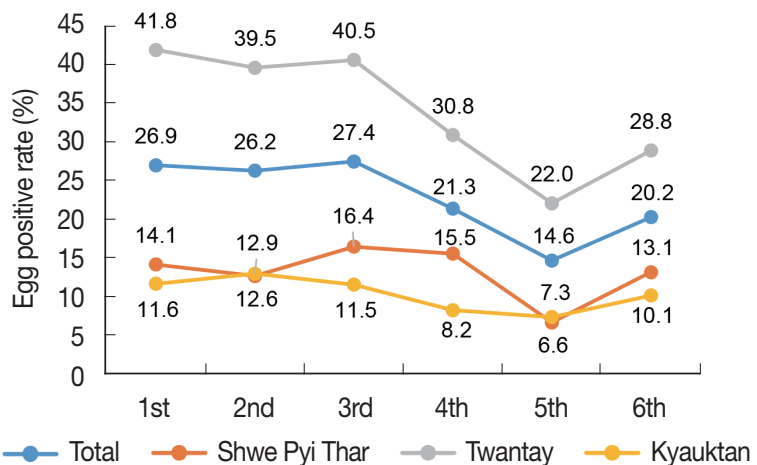

$\mathrm{D}$

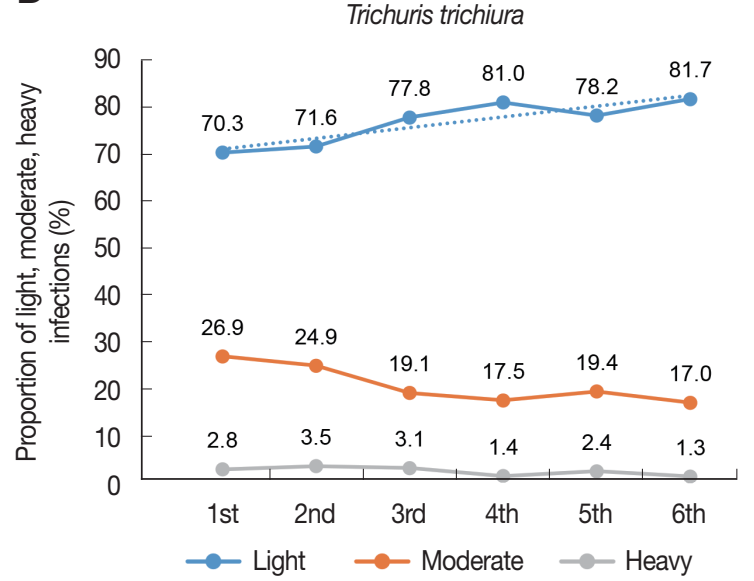

Fig. 3. Prevalence (A, B) and intensity (C, D) of infection with Ascaris lumbricoides and Trichuris trichiura among schoolchildren in the Yangon Region, Myanmar, 2017-2019.

of any STH, A. lumbricoides, and T. trichiura in 3 districts (South Dagon, North Dagon, and Hlaing-thar-yar) of the Yangon Region was $30.9 \%, 17.2 \%$, and $19.4 \%$, respectively. Thus, these districts were classified as the moderate transmission category by WHO (where the prevalence is 20-50\%), and Chai et al. [6] conducted MDA once a year in 2014-2015. Evaluated at 4 months after the 1-time MDA, the prevalence of A. lumbricoides decreased remarkably; however, the prevalence of T. trichiura was not significantly reduced [6].

Another study in Myanmar showed that the reinfection of STH, including A. lumbricoides, T. trichiura, and hookworms, persisted among people in Myanmar, and 6-month gap was time enough for STH prevalence to return to the previous level (baseline prevalence, 27.9\% for any STH) [9]. In the present study, the baseline prevalence of any STH was 37.6\% (28.9\% in Shwe Pyi Thar, 52.8\% in Twantay, 17.3\% in Kyauktan), and we thought that at least MDA twice a year would be necessary for effective control of STH infections in these districts.
We applied a modified scheme of MDA for STH control (especially $T$. trichiura) by changing the frequency of MDA from 2 to 4 times a year. In 2017, MDAs were conducted twice a year using a single dose of albendazole (400 mg). However, the prevalence of T. trichiura persisted (averaged 26.9\% in 3 districts in June 2017, and 26.2\% in November 2017). Thus, in 2018, MDAs were repeated 3 times a year. The results revealed only a slight decrease in the prevalence of $T$. trichiura, being averaged at 21.3\% in November 2018. Moreover, in 2019, despite that MDAs were repeated 4 times a year, the prevalence of $T$. trichiura still remained at average $20.2 \%$ in 3 districts in November 2019, probably due to a lowered drug efficacy and rapid reinfection (Fig. 3B). Meanwhile, the prevalence of $A$. lumbricoides decreased significantly from 23.3\% in June 2017 to $3.6 \%$ in November 2019.

A high coverage rate of MDA in blanket mass chemotherapy is a crucial element that may lead to successful STH control, and we evaluated the coverage rate of each MDA on school- 
children in our project program. The coverage rate gradually increased from $75.1 \%$ in 2017 , to $92.8 \%$ in 2019 , which were higher than the WHO's recommendation of minimum $75 \%$ [8]. Thus, the MDA coverage applied in this study could be justified as adequate for control of STH.

One of the reasons for the failure to reduce T. trichiura prevalence in this study includes possible lowered efficacy of albendazole against this nematode infection [10,11]. In this respect, it is worth to mention that the efficacy profiles of benzimidazole anthelmintics, in particular, albendazole and mebendazole, against $T$. trichiura have shown a decreasing tendency over the past decades [10]. For example, the reported cure rate of albendazole (400 $\mathrm{mg}$ in a single dose) against T. trichiura was $38.6 \%$ (26.2-52.7\%) in 1995 but it decreased to $16.4 \%$ (7.7-31.3\%; $P=0.027)$ in 2015, and the egg reduction rate, which was $72.6 \%$ (53.7-91.5\%) in 1995, decreased to $43.4 \%$ (23.5-63.3\%; $P=0.049)$ in $2015[10,12]$. Mebendazole (500 $\mathrm{mg}$ in a single dose) also showed reduced efficacy against $T$. trichiura; for example, the high egg reduction rate of av. 91.4\% (72.9-100\%) in 1995 was reduced to av. 54.7\% (34.6-74.8\%) in 2015 [10,12].

An important reason for the lowered efficacy of benzimidazoles against trichuriasis is possibly the appearance of drug resistance of worms [10-12]. Drug resistance against benzimidazole anthelmintics has been reported in several human intestinal nematodes, including hookworms ( $N$. americanus), $T$. trichiura, and A. lumbricoides, and it might be related with gene mutation at codon 167, 198 or 200 in the $\beta$-tubulin gene of the worms $[11,13]$. In T. trichiura, mutation at codon 200 was suggested to be related with the drug resistance in Haiti, Kenya, and Panama [13]. Until present, however, no mutation at the $\beta$-tubulin gene of Burmese T. trichiura worms/eggs has been demonstrated in relation to the drug resistance against benzimidazole anthelmintics. Thus, further studies are required in Myanmar to identify single nucleotide polymorphism (SNP) at position 200 or other locus of the $\beta$-tubulin gene of $T$. trichiura in association with the drug resistance.

Another reason for the failure to reduce T. trichiura prevalence in these areas seems to be rapid reinfection. Challenging factors which may be associated with rapid reinfection and sustained prevalence include low socioeconomic status, migrant population, poor sanitary conditions, unsafe water, malnutrition or under-nutrition, and lack of awareness about intestinal parasites, all of which may facilitate the transmission of STH (in particular, T. trichiura) infection and reinfection. We could not perform any measures to improve socioeconomic and environmental conditions in these areas (except for health education), for example, supply of clean water system, change of toilets, prevention from flood, and improvement of sewage system. This may have exerted negative influence on the control of trichuriasis.

In the present study, we performed repeated MDAs in combination with health education to control STH infections among schoolchildren in Myanmar. Health education was performed using interesting audiovisual materials targeting schoolchildren, parents, and residents. Also, various other materials, such as banners, posters, and T-shirts containing health education content were presented to the schoolchildren, guardians, and other participants. However, we regret that the prevalence of T. trichiura is sustained despite all these control programs.

Myanmar has made considerable progress toward controlling STH infections, including A. lumbricoides and hookworms. However, T. trichiura infection remained to be a public health concern especially among schoolchildren in suburban areas of the Yangon Region, Myanmar. STH infections are treated with albendazole (400 $\mathrm{mg}$ in a single dose) or mebendazole (500 $\mathrm{mg}$ in a single dose), but both drugs recently showed lowered efficacy against $T$. trichiura. Therefore, in order to control trichuriasis in Myanmar more effectively, a new MDA strategy, for example, using higher doses of albendazole (2-3 times a day for 2-3 days) or changing the drug into a more effective one, such as oxantel pamoate, seems to be necessary.

\section{ACKNOWLEDGMENTS}

The project team would like to thank the staff in National Health Laboratory, Yangon, Myanmar for their kind assistance in this STH control project. Special thanks to KOICA for providing the grant and technical support for this project (KOICA 2017-045).

\section{CONFLICT OF INTEREST}

The authors declare no conflict of interest related to this work.

\section{REFERENCES}

1. World Health Organization. Bench Aids for the Diagnosis of In- 
testinal Parasites. 2nd ed. Geneva, Switzerland. World Health Organization. 2019.

2. Gabrielli AF, Montresor A, Chitsulo L, Engels D, Savioli L. Preventive chemotherapy in human helminthiasis: theoretical and operational aspects. Trans R Soc Trop Med Hyg 2011; 105: 683693. https://doi.org/10.1016/j.trstmh.2011.08.013

3. Zeng XJ, Jiang WS, Xie SY, Chen YD, Gu XN, Ge J, Hanga CQ, Lia ZJ, Chena H. Effect of integrated control intervention on soiltransmitted helminth infections in Jiangxi province in southeast China. Acta Trop 2019; 194: 148-154. https://doi.org/10.1016/ j.actatropica.2019.04.001

4. Tun A, Myat SM, Gabrielli AF, Montresor A. Control of soil-transmitted helminthiasis in Myanmar: results of 7 years of deworming. Trop Med Int Health 2013; 18: 1017-1020. https://doi.org/10. 1111/tmi.12130

5. Htoon TT, Tun T, Oo KY, Thein W, Tin HH, Chai JY, Yong TS, Sohn WM. Status of infection with soil-transmitted helminths among primary school children in three selected townships of Yangon Region. Myanmar Heal Sci Res J 2015; 27: 221-226.

6. Chai JY, Sohn WM, Hong SJ, Jung BK, Hong S, Cho S, Park JB, Kim IS, Kim S, Lee KH, Jeoung HG, Htoon TT, Tin HH. Effect of mass drug administration with a single dose of albendazole on Ascaris lumbricoides and Trichuris trichiura infection among schoolchildren in Yangon Region, Myanmar. Korean J Parasitol 2020; 58: 195-200. https://doi.org/10.3347/kjp.2020.58.2.195

7. Ministry of Immigration and Population, Myanmar. The 2014 Myanmar Population and Housing Census: Highlights of the
Main Results. Census Report Vol 2. Nay Pyi Taw, Myanmar. Ministry of Immigration and Population, Myanmar. 2015, pp 1-47.

8. World Health Organization. Guideline: Preventive Chemotherapy to Control Soil-Transmitted Helminth Infections in at-Risk Population Groups. Geneva, Switzerland. World Health Organization, 2017.

9. Dunn JC, Bettis AA, Wyine NY, Lwin AMM, Tun A, Maung NS, Anderson RM. Soil-transmitted helminth reinfection four and six months after mass drug administration: results from the delta Region of Myanmar. PLoS Negl Trop Dis 2019; 13: e000659116. https://doi.org/10.1371/journal.pntd.0006591

10. Moser W, Schindler C, Keiser J. Efficacy of recommended drugs against soil transmitted helminths: systematic review and network meta-analysis. BMJ 2017; 358: j4307. http://doi.org/10.1136/bmj. j4307

11. Chai JY, Jung BK, Hong SJ. Albendazole and mebendazole as anti-parasitic and anti-cancer agents: an update. Korean J Parasitol 2021; 59: 1-37. https://doi.org/10.3347/kjp.2021.59.3.189

12. Moser W, Schindler C, Keiser J. Drug combinations against soiltransmitted helminth infections. Adv Parasitol 2019; 103: 91-115. https://doi.org/10.1016/bs.apar.2018.08.002

13. Diawara A, Halpenny C, Churcher TS, Mwandawiro C, Kihara J, Kaplan RM, Streit TG, Idaghdour Y, Scott ME, Basáñez MG, Prichard RK. Association between response to albendazole treatment and $\beta$-tubulin frequencies in soil-transmitted helminths. PLoS Negl Trop Dis 2013; 7: e2247. https://doi.org/10.1371/ journal.pntd.0002247 
